Supplementary information

Size Effect of Atomic Gold Clusters for Carbon Monoxide Passivation at $\mathbf{R u}_{\text {Core }}-\mathbf{P t}_{\text {Shell }}$ Nanocatalysts

Authors: Tsan-Yao Chen, ${ }^{1 *}$ Yu-Ting Liu, ${ }^{2 *}$ Jeng Han Wang, ${ }^{3}$ Guo-Wei Lee, ${ }^{4}$ Po-Wei Yang, ${ }^{1}$ Kuan-Wen Wang ${ }^{5}$

Affiliations:

${ }^{1}$ Department of Engineering and System Science, National Tsing Hua University, Hsinchu 30013, Taiwan Fax: +886 3 5728445; Tel: +886 3 5715131\#34271; E-mail: chencaeser@gmail.com

${ }^{2}$ Department of Soil and Environmental Science, National Chung Hsing University, Taichung 402, Taiwan; E-mail: yliu@nchu.edu.tw

${ }^{3}$ Department of Chemistry, National Taiwan Normal University, Taipei 106, Taiwan

${ }^{4}$ Department of Physics, National Tsing Hua University, Hsinchu 30013, Taiwan

${ }^{5}$ Institute of Materials Science and Engineering, National Central University, Taiwan

*To whom correspondence should be addressed: Tsan-Yao Chen, email:

chencaeser@gmail.com; Tel: +886-3-5742671 (O); +886-3-5728445 (Fax); ${ }^{\S}$ Yu-Ting Liu, email: yliu@nchu.edu.tw 
1. Temperature programmed reduction method

In each TPR analysis, a sample of approximately $20 \mathrm{mg}$ was inserted into a U-shape quartz tube and pre-oxidized in air at room temperature $(300 \mathrm{~K})$ for $1 \mathrm{~h}$. Subsequently, the pre-oxidized catalysts (alloy or reference samples) were reduced by a flow of $20 \%$ $\mathrm{H} 2$ in $\mathrm{N} 2$ at a flow rate of $30 \mathrm{~mL}$ min-1 upon increasing the temperature from 118 to $473 \mathrm{~K}$ at a heating rate of $7 \mathrm{~K} \mathrm{~min}-1$. Once the reduction process was started, the rate of hydrogen consumption presented in the TPR profile was measured automatically by a thermal conductivity detector (TCD). Moreover, silica gel and molecular sieve absorbents were utilized for the purpose of water removal before the flowing gas reached the detector. 
2. XPS analysis on the surface composition of core-shell NCs

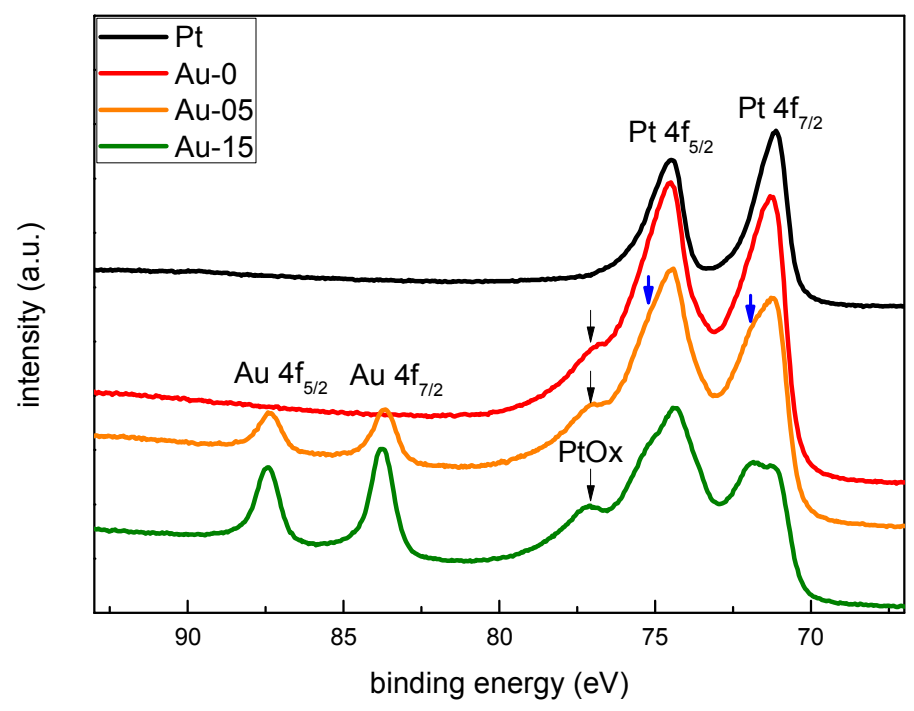

Fig. S1. The Pt $4 \mathrm{f}$ and Au 4f XPS spectra of the Pt NPs, Au-0, Au-05, and Au-15 NPs.

The surface Au to Pt ratios of the NCs was determined to be $0 \%, 7.78 \%$, and $20.05 \%$ for $\mathrm{Au}-0, \mathrm{Au}-5$, and Au15, respectively, by comparing the peak areas with the consideration of the X-ray scattering cross section of the emission lines at selected orbitals. According to Table S1, the emission lines of Au-05 and Au-15 at Pt $4 \mathrm{f}_{5 / 2}$ and $4 \mathrm{f}_{7 / 2}$ orbitals are split into two peaks with the energy shift by 0.71 and $0.63 \mathrm{eV}$ comparing to that of $\mathrm{Au}-0$, respectively. Formation of $\mathrm{PtO}$ is a general consideration for the peak splitting in this region. However, the binding energy shift of $\mathrm{Pt}^{2+}$ species is larger than $1.0 \mathrm{eV}$. Therefore the observed feature can be attributed to the inductive electron relocation by lattice strain or electronegative coupling effects between $\mathrm{Au}$ cluster and the Pt shell crystal. Combining results of DFT calculation (Fig. 5 in manuscript), the substantially suppressed $\mathrm{Pt} 4 \mathrm{f}_{7 / 2}$ emission line by $\sim 33 \%$ can be explained by the electron donation from $\mathrm{Pt}$ to Au domains with increasing $\mathrm{Au} / \mathrm{Pt}$ ratios from 0 to 15 at $\%$. 
Table S1. The Pt 4f XPS peak positions of experimental samples

\begin{tabular}{|c|c|c|c|c|}
\hline sample & phase & orbital & $\mathrm{BE}$ & intensity \\
\hline \multirow[t]{4}{*}{ Pt NPs } & Pt metal & $\mathrm{Pt}_{4} \mathrm{f}_{5 / 2}$ & 74.48 & 135328 \\
\hline & & $\mathrm{Pt}_{4} \mathrm{f}_{7 / 2}$ & 71.12 & 162361 \\
\hline & Pt oxide & $\mathrm{Pt}_{4} \mathrm{f}_{5 / 2}$ & 75.47 & \\
\hline & & $\mathrm{Pt}_{7 / 2}$ & 71.95 & \\
\hline \multirow[t]{4}{*}{$\mathrm{Au}-0$} & Pt metal & $\mathrm{Pt}_{4} \mathrm{f}_{5 / 2}$ & 74.45 & 274196 \\
\hline & & $\mathrm{Pt} 4 \mathrm{f}_{7 / 2}$ & 71.21 & 264219 \\
\hline & Pt oxide & $\mathrm{Pt}_{4} \mathrm{f}_{5 / 2}$ & 76.88 & 125190 \\
\hline & & $\mathrm{Pt}_{7 / 2}$ & NA & \\
\hline \multirow[t]{6}{*}{$\mathrm{Au}-05$} & Pt metal & $\mathrm{Pt} 4 \mathrm{f}_{5 / 2}$ & 74.41 & 236220 \\
\hline & & $\mathrm{Pt}_{4} \mathrm{f}_{5 / 2}-\mathrm{S}$ & 75.12 & 203072 \\
\hline & & $\mathrm{Pt}_{4} \mathrm{f}_{7 / 2}$ & 71.21 & 208865 \\
\hline & & $\mathrm{Pt} 4 \mathrm{f}_{7 / 2}-\mathrm{S}$ & 71.83 & 189234 \\
\hline & Pt oxide & $\operatorname{Pt} 4 f_{5 / 2}$ & 76.89 & 110386 \\
\hline & & $\mathrm{Pt}_{4} \mathrm{f}_{7 / 2}$ & NA & \\
\hline \multirow[t]{6}{*}{$\mathrm{Au}-15$} & Pt metal & $\mathrm{Pt}_{4 / 2}$ & 74.32 & 182314 \\
\hline & & $\mathrm{Pt}_{4} \mathrm{f}_{5 / 2} \mathrm{~S}$ & 75.05 & 156890 \\
\hline & & $\operatorname{Pt} 4 f_{7 / 2}$ & 71.23 & 130018 \\
\hline & & $\mathrm{Pt} 4 \mathrm{f}_{7 / 2}-\mathrm{S}$ & 71.87 & 132753 \\
\hline & Pt oxide & $\mathrm{Pt}_{5 / 2}$ & 77.06 & 92042 \\
\hline & & $\mathrm{Pt}_{4 \mathrm{f}_{7 / 2}}$ & NA & \\
\hline
\end{tabular}


2. Model analysis on Small angle X-ray Scattering spectra of core-shell NCs.

All SAXS data were collected at the In-Achromate superconducting wigglers (IASW) beamline at BL-23A of the National Synchrotron Radiation Research Center (NSRRC, Taiwan) with incident X-ray beam at a wavelength of $0.82667 \AA$ (15.0 keV). The SAXS data were corrected for sample transmission, background, and detector sensitivity, and normalized to the absolute scattering scale, namely, scattering cross-section per unit sample volume $I(q)$. Samples (containing $\sim 0.1 \mathrm{mg}$ of NPs and $\sim 1.0 \mathrm{wt} \%$ of PVP-40 in distilled water) were sealed in a $1.0 \mathrm{~mm}$ thick stainless steel cell using two kapton films ( $\sim 25 \mu \mathrm{m}$ thick) as the X-ray windows. With the sample to - detector distance was set to be $2427.6 \mathrm{~mm}$. The SAXS data were collected by an area detector (Pilatus 1M-F, $7 \mathrm{~ms} /$ frame, $169 \mu \mathrm{m}$ by $179 \mu \mathrm{m}$ ) covering $q$-range from 0.01 to $0.4 \AA^{-1}$. The obtained scattering functions were analyzed using a to component SAXS model comprising fractal aggregates ${ }^{1,2}$ and core-shell NPs with the external factor of $\mathrm{Au}$ clusters at the scattering matter ${ }^{3}$. By considering a suspension of spherically symmetric core-shell particles with polydispersion; the scattering intensity can be expressed as the combination of the contributions from the fractal aggregate and the core-shell particle as shown in Equation (S1):

$$
I(q)=A^{F} \cdot S(q)^{F} \cdot P(q)^{F}+A^{C S_{-} \mathrm{C}} \cdot S(q)^{C S_{-} \mathrm{C}} \cdot P(q)^{C S_{-} \mathrm{C}}
$$

where $A$ is the number density of the scattering objects. Both the $S(Q)$ and the $P(Q)$ are the function of the momentum transfer $(Q)$ between incident and scattered X-ray. $Q$ is given by $Q=(4 \pi / \lambda) \times \sin (\theta / 2)$ in an elastic scattering process; where $\theta$ denotes the angle between the incident and the scattered beams at a wavelength of $\lambda$. The structure factor $S(q)$ and the particle form factor $P(q)$ both depend on the momentum transfer $q$. The superscript symbols of $F$ (fractal aggregate) and CS_C (core-shell cylinder particle) refer to the identity of the parameter belongs to. $P(q)^{F}$ is the scattering from randomly distributed spherical "building block" particles, having radius $R_{0}$, volume fraction $\varphi$, and scattering length density difference $\Delta \rho=\rho_{1}-\rho_{2}$.

$$
P(q)=\phi V_{P} \Delta \rho^{2} F\left(q R_{0}\right)^{2} \ldots(\mathrm{S} 1 \mathrm{~b})
$$

where $V_{P}=(4 / 3) \pi \mathrm{R}_{0}^{3}$ and

$$
F(x)=\frac{3[\sin (x)-x \cos (x)]}{x^{3}} \ldots(\mathrm{S} 1 \mathrm{c})
$$

The structure factor $S(q)^{F}$ is calculated as:

$$
S(q)=1+\frac{\sin \left[\left(D_{f}-1\right) \tan ^{-1}(q \xi)\right]}{\left(q R_{0}\right)^{D_{f}}} \frac{D_{f} \Gamma\left(D_{f}-1\right)}{\left[1+1 /\left(q^{2} \xi^{2}\right)\right]^{\left(D_{f}-1\right) / 2}} \ldots(\mathrm{S} 1 \mathrm{a})
$$

where $\xi$ corresponds to the overall coherent length (size) of aggregates, $D_{f}$ is the 
self-similarity packing dimension of nanoparticles in aggregates, and $R_{0}$ is the radius of randomly distributed spherical "building block" particles in the aggregate. $\Gamma\left(D_{f}-1\right)$ is a gamma function.

Considering that the particle has a core-shell architecture, the form factor $P(q)^{\mathrm{CS}}$ should be written as:

$$
P(q)=\frac{\text { scale }}{V_{S}}\left[\frac{3 V_{C}\left(\rho_{C}-\rho_{S}\right) j_{1}\left(q r_{C}\right)}{q r_{C}}+\frac{3 V_{S}\left(\rho_{S}-\rho_{\text {Solv }}\right) j_{1}\left(q r_{S}\right)}{q r_{S}}\right]^{2}+b k g \ldots(\text { S }
$$

where $j_{1}(x)=(\sin x-x \cos x) / x^{2}, r_{\mathrm{S}}=r_{\mathrm{C}}+\mathrm{t}$ and $V_{i}=(4 \pi / 3) r_{i}^{3}$ with respective to the geometry parameters given in below:

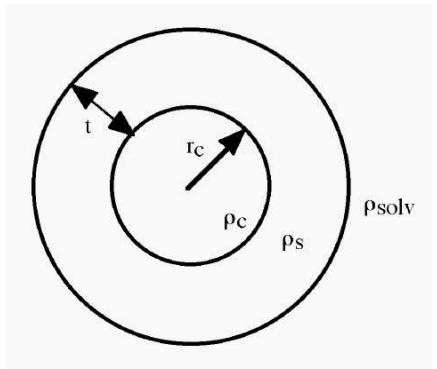

Reference: Guinier, A. and G. Fournet, "Small-Angle Scattering of X-Rays", John Wiley and Sons, New York, (1955).

With the constrain of polydispersity of core $\left(P=\sigma / r_{\mathrm{C}}\right.$, where $\sigma^{2}$ is the variance of the distribution and $r_{\mathrm{C}}$ is the mean core radius) the returned form factor should be normalized by the average particle volume $\langle V\rangle$ :

$$
\langle V\rangle=\frac{4 \pi}{3}\left\langle r^{3}\right\rangle \text { where }\left\langle r^{3}\right\rangle=\frac{(z+3)(z+2)}{(z+1)^{2}}\langle r\rangle
$$

and $z$ is the width parameter of the Schultz distribution, $z=\left(1 / \rho^{2}\right)-1$.

Whereas, the scattering patterns of core-shell NPs intercalated by Au clusters were fitted using a model modified from core-shell cylinder. The inter-particle scattering correlation factors $\left(A^{F}, S(Q)^{F}\right.$, and $\left.P(Q)^{F}\right)$ are modified into the inter-cluster ones and represented by $A^{F c l u}, S(Q)^{F c l u}$, and $P(Q)^{F c l u}$ function shown in Equation (S1).

2. Temperature programmed reduction analysis

In each temperature programmed reduction(TPR)analysis, a sample of approximately $20 \mathrm{mg}$ was inserted into a U-shape quartz tube and pre-oxidized in air 
at room temperature $(300 \mathrm{~K})$ for $1 \mathrm{~h}$. Subsequently, the pre-oxidized catalysts (alloy or reference samples) were reduced by a flow of $20 \% \mathrm{H} 2$ in $\mathrm{N} 2$ at a flow rate of 30 $\mathrm{mL}$ min-1 upon increasing the temperature from 118 to $473 \mathrm{~K}$ at a heating rate of $7 \mathrm{~K}$ min-1. Once the reduction process was started, the rate of hydrogen consumption presented in the TPR profile was measured automatically by a thermal conductivity detector (TCD). Moreover, silica gel and molecular sieve absorbents were utilized for the purpose of water removal before the flowing gas reached the detector. 
3. The configuration for atomic packing of Au clusters at Rucore-Ptshell nanoparticles

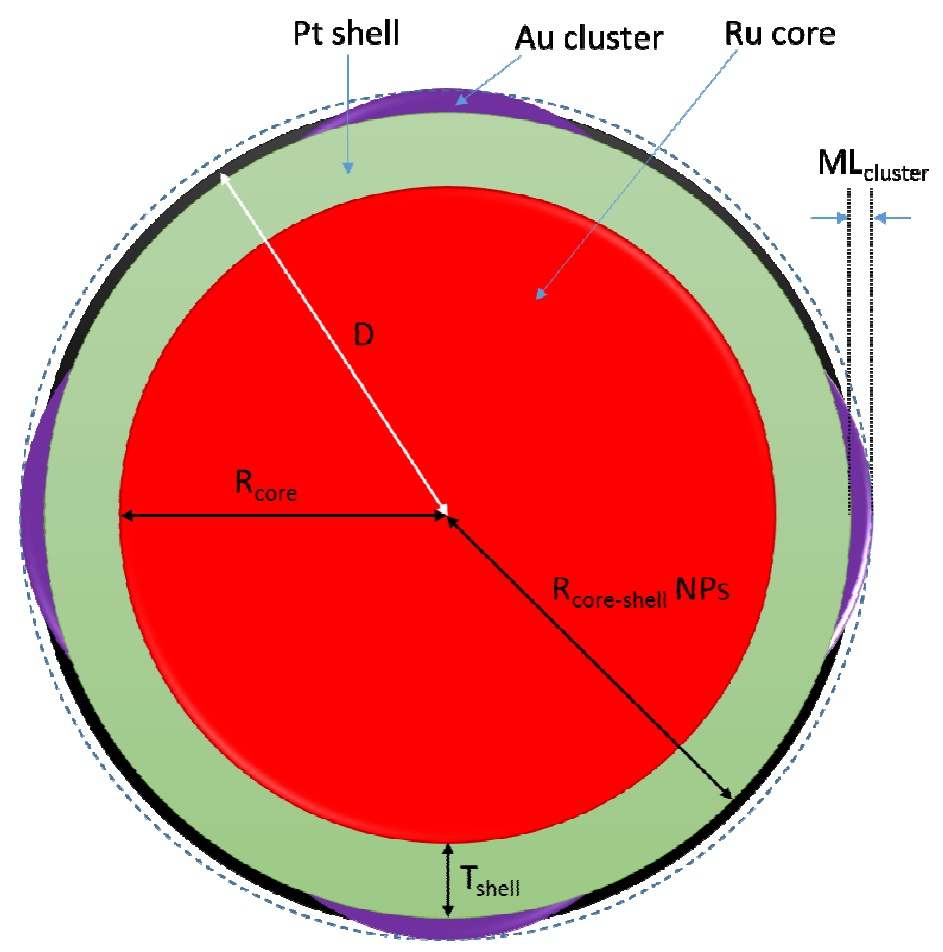

Scheme S1. The configuration for atomic packing of Au clusters at Rucore-Ptshell nanoparticles. $\mathrm{ML}_{\text {cluster: }}$ atomic monolayers of cluster; ø: coverage of cluster atop the core-shell NPs; $\mathrm{R}_{\text {core-shell }} \mathrm{NPs}$ : the radius of core-shell NPs; $\mathrm{T}_{\text {shell: }}$ thickness of shell crystal; $\mathrm{R}_{\text {core }}$ : radius of core crystal

The geometrical configuration of core-shell nanoparticles intercalated with $\mathrm{Au}$ cluster is shown in Scheme S1; where the numerical correlations between the structure parameters are interpreted as following:

Final particle diameter (Df)

$=\mathrm{D}_{\text {core-shell }} \mathrm{NPs}+2 \times\left(\varnothing \mathrm{ML}_{\text {cluster }}\right)$

$=2 \times\left(\mathrm{R}_{\text {core-shell }} \mathrm{NPs}+\varnothing \mathrm{ML}_{\text {cluster }}\right)$

$\rightarrow$ Growth of $\mathrm{D}=\mathrm{Df}-\mathrm{D}_{\text {core-shell }} \mathrm{NPs}=2 \mathrm{x}+\varnothing \mathrm{Ml}_{\text {cluster }}$

$\rightarrow$ Growth of $D=2 \times 0.25 \times 0.3 \mathrm{~nm}=0.15 \mathrm{~nm}$ 


\section{XAS analysis}

Atomic structures of electrocatalysts were characterized by X-ray absorption spectroscopy. The Pt L3-edge (11564 eV) and Ru K-edge (22117 eV) X-ray absorption spectra (XAS) were collected by fluorescence mode (EXAFS) using a conventional Soler-slit Lytle detector with appropriate filters (Ge and Mo for $\mathrm{Pt}$ L3-edge and $\mathrm{Ru} \mathrm{K}$-edge, respectively) at the Wiggler beam line BL-17C and the superconducting wavelength shifter beam line BL-01C1 of the National Synchrotron Radiation Research Center (NSRRC, Taiwan), respectively.
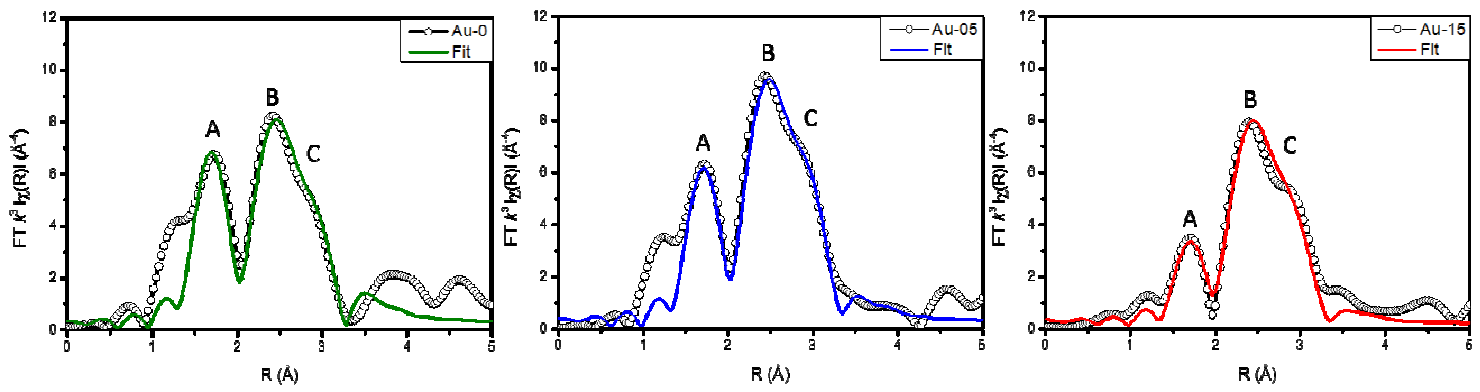

Fig. S2 The Pt $\mathrm{L}_{3}$-edge Fourier transformed EXAFS function with the fitting curves of Au- $0, \mathrm{Au}-05$, and $\mathrm{Au}-15 \mathrm{NCs}$.

The Pt L3-edge Fourier transformed EXAFS spectra (radial structure function, RSF) with the fitting curves of the Au-0, Au-05, and Au-15 NCs are demonstrated in Fig. S4. In these RSF curves, the three radial peaks A, B, C are contributions of the $\mathrm{X}$-ray interferences between the bond pairs of Pt-O, Pt-PtM/Pt-Ptox1, and Pt-Ptox2, respectively (where Pt-PtM and Pt-Ptox1/Pt-Ptox2 denote the bond pair in metallic $\mathrm{Pt}$ and Pt oxide domains). The shoulder at the left hand side of peak A can be attributed to the abnormal photoelectron convolution results due to the co-existence of strong out of phase waves between Pt and Pt oxide domains. As can be seen, all the experiment spectra were well fitted by the model simulations.

The Ru K-edge RSF spectra of the Au-0, Au-05, and Au-15 NCs (annealed at $330^{\circ} \mathrm{C}$ for $30 \mathrm{mins}$ ) are compared in Fig. S3. As indicated, the two radial peaks A and $\mathrm{B}$ are contributions of $\mathrm{Ru}-\mathrm{O}$ and $\mathrm{Ru}-\mathrm{Ru}$ bond pairs in oxide and metal phases, respectively. For Au-0 NCs, the considerable intensity of peak A could be accounted to the slight oxidation at the core-shell interface where certain amount of Ru atoms were exposed to the outmost layer due to the relocation of Pt shell atoms during annealing. Increasing the amount of Au clusters suppresses the Ru oxidation which again proving their shielding effects at the NCs. 


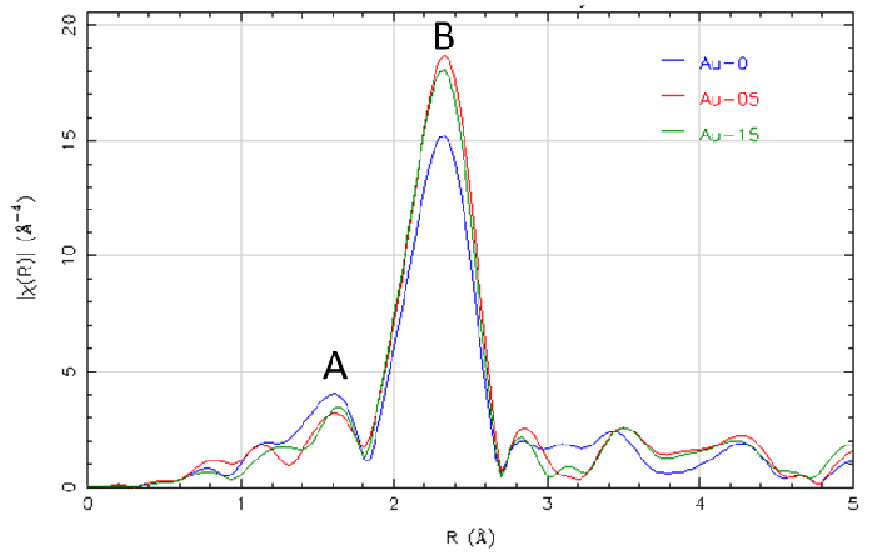

Fig. S3 The Ru K-edge RSF spectra of Au-0, Au-05, and Au-15 NCs. 
5. DFT calculation results of $\mathrm{O}$ and $\mathrm{CO}$ binding energy at different sorption sites
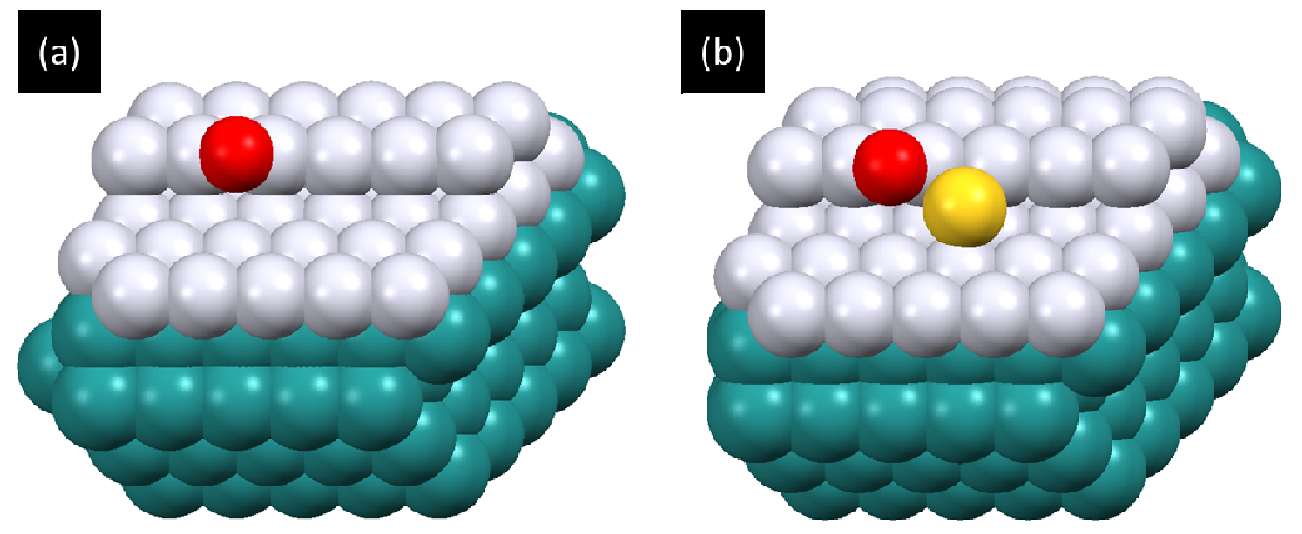

Fig. S4. The atomic model of oxygen chemisorption atop the bridge site of Pt shell edge on (a) Ru (0001) 2x2 + 1.5ML Pt + Oads(c1) and (b) Ru (0001) 2x2 + 1.5ML Pt $+\mathrm{Au}(\mathrm{H} 1$ site $)+$ Oads(c1) models. 


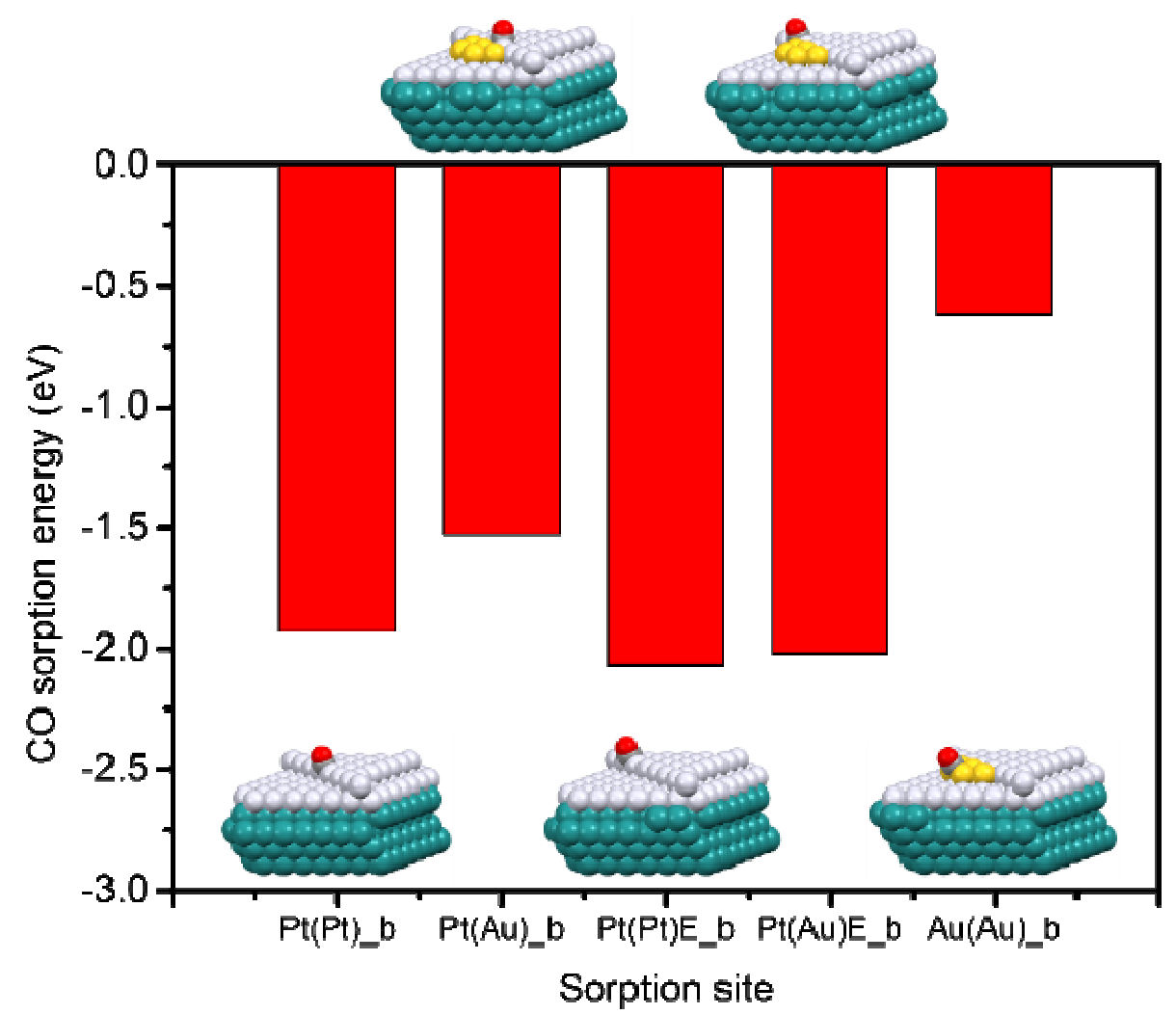

Fig. S5 DFT calculation results of CO sorption model on 1.5 MLs of Pt atoms on the $\mathrm{Ru}(0001)$ facet with and without $\mathrm{Au}$ clusters in the kink region with the corresponding sorption energies. ${ }^{2}$

The binding energies of chemisorbed $\mathrm{CO}$ at $\mathrm{Au}$ atom and $\mathrm{Pt}$ atoms in topmost surface of Rucore-Ptshell NCs are substantially lower than that at the corner sites (see Fig. S5). This is the reason that only the sorption features of $\mathrm{CO}$ at edge (corner) sites are discussed in this paper. 
6. The DFT calculation determined work function shift on Ru(0001)@Pt w/ and w/o Au intercalation.

According to the DFT results in Fig. 6, the Au intercalation will redistribute the electron density of Ru(0001)@Pt model. It triggers the negative charge localization at the near surface region. For confirming the effects of gold intercalation on the oxygen binding energy and the work function of the core-shell nanoparticle surface were calculated by DFT and results in Table S2. As indicated, the chemisorption energy of oxygen $\left(\mathrm{O}^{\text {ads }} \mathrm{EB}\right)$ at the $\mathrm{c} 1$ site (Fig. S4, the bridge site at the edge of shell crystal) is substantially reduced by $0.20 \mathrm{eV}$ (from 6.26 to $6.20 \mathrm{eV}$ ) by the presence of Au atom in the neighboring hollow site. Such phenomenon could be attributed to the charge extraction from the substrate to these regions as a result of the strong electronegativity and the local lattice strain of the gold atom intercalation. The electron extraction is further illustrated by the reduction of work function (Wf) by $0.112 \mathrm{eV}$ (from 4.909 to $4.797 \mathrm{eV}$ ) in the presence of $\mathrm{Au}$ atom at the edge bridge site. This feature suggests the decreasing energy barrier for ejecting the electron from the bulk surface to the continuum or vacuum states by the presence of strong local electron repulsive fields (electron rich region) around the gold atoms. In this circumstance, this electron repulsive fields substantially suppress the $\mathrm{CO}$ poisoning of NCs. The CO sorption 
energy $\left(\mathrm{CO}^{\mathrm{ads}} \mathrm{E}\right)$ at different sorption sites are compared in Fig. $\mathbf{S 5}$ and in previous work. In our study, the $\mathrm{CO}^{\mathrm{ads}} \mathrm{E}$ of chemisorbed $\mathrm{CO}$ at bridge sites between (1) $\mathrm{Pt}$ atoms at slab surface (Pt(Pt)_b), (2) Au and Pt atoms at slab surface (Pt(Au)_b), (3) at Pt atoms at slab edge $\left(\mathrm{Pt}(\mathrm{Pt}) \mathrm{E} \_b\right)$, (4) Pt atom at the slab edge with Au cluster $\left(\operatorname{Pt}(\mathrm{Au}) \mathrm{E}_{-} \mathrm{b}\right)$, and $\mathrm{Au}$ atoms on the cluster $\left(\mathrm{Au}(\mathrm{Au}) \_b\right)$ are determined to be -1.93, $-1.53,-2.07,-2.02$, and $-0.62 \mathrm{eV}$, respectively. Among these sorption sites, the highest $\mathrm{CO}^{\mathrm{ads}} \mathrm{E}$ of $\mathrm{Pt}(\mathrm{Pt}) \_\mathrm{E}$ indicates its strongest preference for $\mathrm{CO}$ chemisorption. This phenomenon could be rationalized by the strong chemisorption at edge sites that eliminated the defect density the most among presenting models in order to minimize the surface energy. It is important to note that, the $\mathrm{E}^{\text {ads }} \mathrm{CO}$ at sorption sites containing $\mathrm{Au}$ atom $\left(\mathrm{Pt}(\mathrm{Au}) \_\mathrm{b}\right.$ and $\left.\mathrm{Au}(\mathrm{Au}) \_b\right)$ are substantially suppressed by 0.4 to $1.45 \mathrm{eV}$ comparing to that of sites without $\mathrm{Au}$ participant $\left(\mathrm{Pt}(\mathrm{Pt}){ }_{-} \mathrm{b}, \mathrm{Pt}\left(\mathrm{PtE} \_\mathrm{b}\right)\right.$, and $\operatorname{Pt}(\mathrm{Au}) \mathrm{E}_{-}$b). This result, consistent with the X-ray photoemission analysis (Fig. S1, Table S1 and Fig. 5) and DFT calculation (Fig. 6), explains the strong CO passivation by the electron localization due to the local lattice strain and the electronegative dipole at the heterojunction interface between Au cluster and Pt bulk. 
Table S2. The DFT calculated structure parameters of bimetallic NPs.

\begin{tabular}{ccccc}
\hline Model & Slab configuration & $\mathrm{Efinal}^{(\mathrm{eV})^{a}}$ & $\begin{array}{c}\mathrm{O}^{\mathrm{ads}} \\
\mathrm{EB}(\mathrm{eV})^{b}\end{array}$ & $\begin{array}{c}\mathrm{Wf} \\
(\mathrm{eV})^{c}\end{array}$ \\
\hline $\mathrm{Ru}(0001) @ \mathrm{Pt}$ & $\mathrm{Ru}(0001) 2 \times 2+1.5 \mathrm{ML} \mathrm{Pt}$ & -700.613 & $\mathrm{NA}$ & 4.909 \\
& $\mathrm{Ru}(0001) \begin{array}{c}2 \times 2+1.5 \mathrm{ML} \mathrm{Pt}+ \\
\mathrm{O}^{\mathrm{ads}}(\mathrm{c} 1)\end{array}$ & -706.753 & 6.26 & 5.192 \\
\hline $\mathrm{Ru}(0001) @ \mathrm{Pt}-\mathrm{Au}$ & $\begin{array}{c}\mathrm{Ru}(0001) 2 \times 2+1.5 \mathrm{ML} \mathrm{Pt}+ \\
\mathrm{Au}(\mathrm{H} 1 \text { site) }\end{array}$ & -703.547 & $\mathrm{NA}$ & 4.797 \\
& $\begin{array}{c}\mathrm{Ru}(0001) 2 \times 2+1.5 \mathrm{ML} \mathrm{Pt}+ \\
\mathrm{Au}(\mathrm{H} 1 \mathrm{site})+\mathrm{O}^{\mathrm{ads}}(\mathrm{c} 1)\end{array}$ & -709.988 & 6.06 & 5.163 \\
\hline
\end{tabular}

